\title{
Aminopropyltriethoxysilane-mediated surface functionalization of hydroxyapatite nanoparticles: synthesis, characterization, and in vitro toxicity assay
}

\author{
This article was published in the following Dove Press journal: \\ International Journal of Nanomedicine \\ 16 December 2011 \\ Number of times this article has been viewed
}

\section{Shige Wang' \\ Shihui Wen ${ }^{2}$ \\ Mingwu Shen ${ }^{2}$ \\ Rui Guo 2 \\ Xueyan $\mathrm{Cao}^{2}$ \\ Jianhua Wang ${ }^{3}$ \\ Xiangyang Shi ${ }^{1,2,4}$}

'State Key Laboratory for Modification of Chemical Fibers and Polymer Materials, ${ }^{2}$ College of Chemistry, Chemical Engineering and Biotechnology, Donghua

University, ${ }^{3}$ Department of Biochemistry and Molecular Cell Biology, School of Medicine, Shanghai Jiao Tong University, Shanghai, People's Republic of China; ${ }^{4}$ Centro de Química da Madeira, Universidade da Madeira, Campus da Penteada, Funchal, Portugal

Correspondence: Xiangyang Shi 2999 North Renmin Road, College of Chemistry, Chemical Engineering and Biotechnology, Donghua University, Shanghai 201620, People's Republic of China

Tel +86 2I 67792656

Fax +86 2I 67792306804

Email xshi@dhu.edu.cn

Jianhua Wang

Department of Biochemistry and Molecular Cell Biology, Shanghai Jiao Tong University School of Medicine, Shanghai 200025, People's Republic of China

Tel +86 2l 5466087 I

Fax +86 2I 63842I57

Email jianhuaw2007@gmail.com
Background: We report on aminopropyltriethoxysilane (APTS)-mediated surface modification of nanohydroxyapatite with different surface functional groups for potential biomedical applications. In this study, nanohydroxyapatite covalently linked with APTS (n-HA-APTS) was reacted with acetic anhydride or succinic anhydride to produce neutralized (n-HA-APTS. Ac) or negatively charged (n-HA-APTS.SAH) nanohydroxyapatite, respectively. Nanohydroxyapatite formed with amine, acetyl, and carboxyl groups was extensively characterized using Fourier transform infrared spectroscopy, transmission electron microscopy, ${ }^{1} \mathrm{H}$ nuclear magnetic resonance spectroscopy, X-ray diffraction, inductively coupled plasma-atomic emission spectroscopy, and zeta potential measurements.

Results: In vitro 3-(4,5-dimethylthiazol-2-yl)-2,5-diphenyltetrazolium bromide colorimetric assay revealed that the slight toxicity of the amine-functionalized n-HA-APTS could be eliminated by post-functionalization of APTS amines to form acetyl and carboxyl groups. Blood compatibility assessment demonstrated that the negligible hemolytic activity of the pristine nanohydroxyapatite particles did not appreciably change after APTS-mediated surface functionalization.

Conclusion: APTS-mediated functionalization of nanohydroxyapatite with different surface groups may be useful for further functionalization of nanohydroxyapatite with biologically active materials, thereby providing possibilities for a broad range of biomedical applications.

Keywords: aminopropyltriethoxysilane, hydroxyapatite nanoparticles, surface functionalization, acetylation, carboxylation, toxicity

\section{Introduction}

Recent advances in nanotechnology have shown that inorganic nanoparticles have attracted immense scientific and technological interest in the areas of catalysis, ${ }^{1,2}$ sensors, ${ }^{3,4}$ molecular imaging, ${ }^{5-8}$ tissue engineering, ${ }^{9}$ and drug delivery. ${ }^{10}$ This is largely due to their nanometer size, unique chemical and physical properties, and tailored surface functionality. Nanohydroxyapatite with the structural formula of $\mathrm{Ca}_{10}\left(\mathrm{PO}_{4}\right)_{6}(\mathrm{OH})_{2}$ is the principal inorganic ingredient of bone and teeth of the human body. ${ }^{11}$ Studies from laboratory research on therapeutic applications have demonstrated that nanohydroxyapatite has a similar crystallographic structure to that of natural bone and excellent biological characteristics, such as nonimmunogenicity, noninflammatory behavior, good biocompatibility, and high osteoconductivity and osteoinductivity. ${ }^{12,13}$ These distinct features enable a wide range of potential applications of nanohydroxyapatite 
as a gene carrier, ${ }^{14}$ and in bone tissue engineering, ${ }^{15}$ drug delivery, ${ }^{16}$ and orthopedic surgery. ${ }^{17}$

As a structural material, nanohydroxyapatite possesses some inherent imperfections, such as brittleness, ${ }^{18-21}$ difficulties in surface functionalization, ${ }^{22}$ and weakened colloidal stability in a nanocrystal form. ${ }^{23}$ Consequently, much effort has been devoted to improving the dispersity and colloidal stability of nanohydroxyapatite and promoting the mechanical durability. ${ }^{12,24-30}$ Most of these studies have involved incorporating nanohydroxyapatite into a polymer matrix to form organic/inorganic hybrid composites in the form of membranes ${ }^{12,24,25}$ or nanofibers, ${ }^{26,27}$ However, these composites prepared by simply mixing nanohydroxyapatite with a polymer matrix usually lack strong molecular interaction between organic and inorganic phases which may give rise to compromised processing ability and structural damage to nanohydroxyapatite in some cases. ${ }^{31}$ Hence, finding an approach to modifying or functionalizing nanohydroxyapatite without compromising its physicochemical properties is of vital importance for its biomedical applications.

Aminopropyltriethoxysilane (APTS) is a common silane agent with amino groups that can be further functionalized with general bioconjugation techniques. ${ }^{32}$ In an aqueous environment, APTS can be hydrolyzed to form silanol groups, which are able to react with hydroxyl groups onto particle surfaces or planar surfaces. For instance, magnetic $\mathrm{Fe}_{3} \mathrm{O}_{4}$ and $\mathrm{TiO}_{2}$ nanoparticles with surface hydroxyl groups can be successfully modified with APTS. ${ }^{33-36}$ In a recent study, Balasundaram et $\mathrm{al}^{37}$ reported the surface grafting of hydroxyapatite compacts with APTS for further conjugation with an arginine-glycine-aspartic acid (RGD) peptide sequence for bone tissue engineering. In their study, the APTS aminemodified hydroxyapatite compacts were able to be activated by N-succinimidyl-3-maleimido propionate for RGD peptide conjugation. That study clearly indicates that hydroxyapatite with a hydroxyl surface is able to be modified with APTS for further bioconjugation. It is reasonable to hypothesize that single nanohydroxyapatite particles are also able to be modified in a similar way, thereby providing many opportunities for their further biomedical applications.

In the present study, we attempted to modify nanohydroxyapatite with APTS, and then the APTS amine groups on the surface of nanohydroxyapatite were further modified with acetic anhydride and succinic anhydride to generate surface charge neutral and negatively charged nanohydroxyapatite particles, respectively. The functionalized nanohydroxyapatite derivatives were extensively characterized using Fourier transform infrared spectroscopy (FTIR), transmission electron microscopy (TEM), ${ }^{1} \mathrm{H}$ nuclear magnetic resonance (NMR), X-ray diffraction, inductively coupled plasma-atomic emission spectroscopy (ICP-AES), and zeta potential measurement. The cytotoxicity of these functionalized nanohydroxyapatite derivatives was tested by an MTT (3-(4, 5-dimethylthiazol-2-yl)-2, 5-diphenyltetrazolium bromide) colorimetric assay of mouse fibroblasts (L929 cells). The blood compatibility of these functionalized nanohydroxyapatite derivatives was assessed by in vitro hemolysis assay. To our knowledge, this is the first report related to the modification of nanohydroxyapatite with APTS (n-HA-APTS) and the systematic modification of n-HA-APTS to generate nanohydroxyapatite with different surface charges. We anticipate that the findings from this study will be beneficial for the development of nanohydroxyapatite-based composite materials for various biomedical applications.

\section{Methods and materials Materials}

Nanohydroxyapatite was purchased from Aladdin Chemical Reagent Co, Ltd (Shanghai, China). APTS and MTT were obtained from $\mathrm{J}$ and $\mathrm{K}$ Chemical Co, Ltd (Shanghai, China). Acetic anhydride and succinic anhydride were from Sigma-Aldrich (St Louis, MO). L929 cells were from the Institute of Biochemistry and Cell Biology, the Chinese Academy of Sciences (Shanghai, China). Dulbecco's modified Eagle's medium, fetal bovine serum, penicillin, and streptomycin were purchased from Hangzhou Jinuo Biomedical Technology (Hangzhou, China). All other chemicals were from Sinopharm Chemical Reagent Co, Ltd (Shanghai, China) and used as received. Water used in all experiments was purified using a Milli-Q Plus 185 water purification system (Millipore, Bedford, MA) with resistivity higher than $18 \mathrm{M} \Omega . \mathrm{cm}$.

\section{Modification of nanohydroxyapatite by APTS}

As shown in Scheme 1, nanohydroxyapatite was treated with APTS to introduce amino groups on its surface. Briefly, nanohydroxyapatite $(180 \mathrm{mg})$ was dispersed into ethanol $(200 \mathrm{~mL})$. The mixture was sonicated for 2 hours until a homogeneous solution was obtained. Subsequently, APTS $(5 \mathrm{~mL})$ was added into the nanohydroxyapatite solution under vigorous magnetic stirring. In order to facilitate the silanization reaction, $1 \mathrm{~mL}$ of water was added into the nanohydroxyapatite suspension to promote hydrolysis of the alkoxysilane groups of APTS to form silanol groups. ${ }^{38}$ 
The mixture was allowed to react with stirring for 12 hours at room temperature. After that, the resulting solution was washed with water two times and then with methanol five times to remove the excess reactants and byproducts, followed by centrifugation (6000 rpm, 5 minutes) and air dried to obtain n-HA-APTS.

\section{Acetylation of surface amines of $n$-HA-APTS}

To neutralize the positive charges of the amine-surfaced n-HA-APTS particles, the particles were reacted with acetic anhydride (Scheme 1). The acetylation reaction was performed according to a procedure described in our previous work. ${ }^{39}$ In brief, n-HA-APTS (50 mg) was dispersed thoroughly in dimethylsulfoxide $(15 \mathrm{~mL})$ under magnetic stirring, followed by addition of triethylamine $(1 \mathrm{~mL})$ and the solution was well mixed for 30 minutes. Excess acetic anhydride $(1 \mathrm{~mL})$ dissolved in dimethylsulfoxide $(5 \mathrm{~mL})$ was then added dropwise into the n-HA-APTS/triethylamine mixture solution under vigorous magnetic stirring. The mixture was allowed to react with stirring for 24 hours. Acetylated n-HA-APTS (n-HA-APTS.Ac) particles were obtained using similar washing and centrifugation steps that were used to purify n-HA-APTS particles.

\section{Carboxylation of surface amines of $n$-HA-APTS}

To modify the amine groups of n-HA-APTS particles with carboxyl groups, the n-HA-APTS particles were reacted with succinic anhydride according to a procedure described in our previous report (Scheme 1). ${ }^{39}$ Briefly, n-HA-APTS $(50 \mathrm{mg})$ dispersed in dimethylsulfoxide $(20 \mathrm{~mL})$ was added with excess succinic anhydride $(500 \mathrm{mg})$ dissolved in $5 \mathrm{~mL}$ of dimethylsulfoxide under vigorous magnetic stirring. The reaction was stopped after 24 hours. Thereafter, the carboxylated n-HA-APTS (n-HA-APTS.SAH) formed was purified according to the procedure used to purify n-HA-APTS.

\section{Characterization techniques}

FTIR was performed using a Nicolet Nexus 670 FTIR (Thermo Electron Corporation, Waltham, MA) spectrometer. All spectra were recorded in transmission mode ranging from 650 to $4000 \mathrm{~cm}^{-1}$ under ambient conditions. ${ }^{1} \mathrm{H}$ NMR spectra were recorded on a Bruker AV 400 NMR spectrometer. Samples were dispersed in $\mathrm{D}_{2} \mathrm{O}$ before measurements. Zeta potential measurements were carried out using a Zetasizer Nano ZS system (Malvern, Worcestershire, UK) equipped with a standard $633 \mathrm{~nm}$ laser. Samples were dispersed in water at a concentration of $1 \mathrm{mg} / \mathrm{mL}$ before measurement. TEM was performed using a Jeol 2010F analytical electron microscope (Jeol, Tokyo, Japan) operating at $200 \mathrm{kV}$. A $5 \mu \mathrm{L}$ aqueous solution $(1 \mathrm{mg} / \mathrm{mL})$ of each sample was dropped onto a carbon-coated copper grid and air-dried before measurement. The crystalline structure of nanohydroxyapatite before and after modification was analyzed by a Rigaku D/max-2550 PC X-ray diffraction system (Rigaku Co, Tokyo, Japan) using $\mathrm{Cu} \mathrm{K} \alpha$ radiation with a wavelength of $0.154 \mathrm{~nm}$ at $40 \mathrm{kV}$ and $200 \mathrm{~mA}$. The scan was performed from $5^{\circ}$ to $70^{\circ}(2 \theta)$. The elemental composition of calcium and silicon in the APTS-modified nanohydroxyapatite sample was determined by a Leeman Prodigy ICP-AES system (Hudson, NH03051). A n-HA-APTS sample (1.15 mg) was dissolved into $5 \mathrm{~mL}$ of concentrated nitric acid before measurement.

\section{Cell biology evaluation}

L929 cells were cultured in $25 \mathrm{~cm}^{2}$ tissue culture flasks with $5 \mathrm{~mL}$ Dulbecco's modified Eagle's medium containing $10 \%$ fetal bovine serum, $100 \mathrm{U} / \mathrm{mL}$ penicillin, and $100 \mathrm{U} / \mathrm{mL}$ streptomycin in an incubator with $5 \% \mathrm{CO}_{2}$ at $37^{\circ} \mathrm{C}$. Cells $\left(1 \times 10^{4}\right.$ cells per well $)$ were plated into a 96-well plate in a complete medium overnight. The medium was replaced by fresh medium containing samples with a concentration range of $0-100 \mu \mathrm{g} / \mathrm{mL}$. After 24 hours of incubation, an MTT assay was used to quantify the viability of the cells according to the manufacturer's instructions.

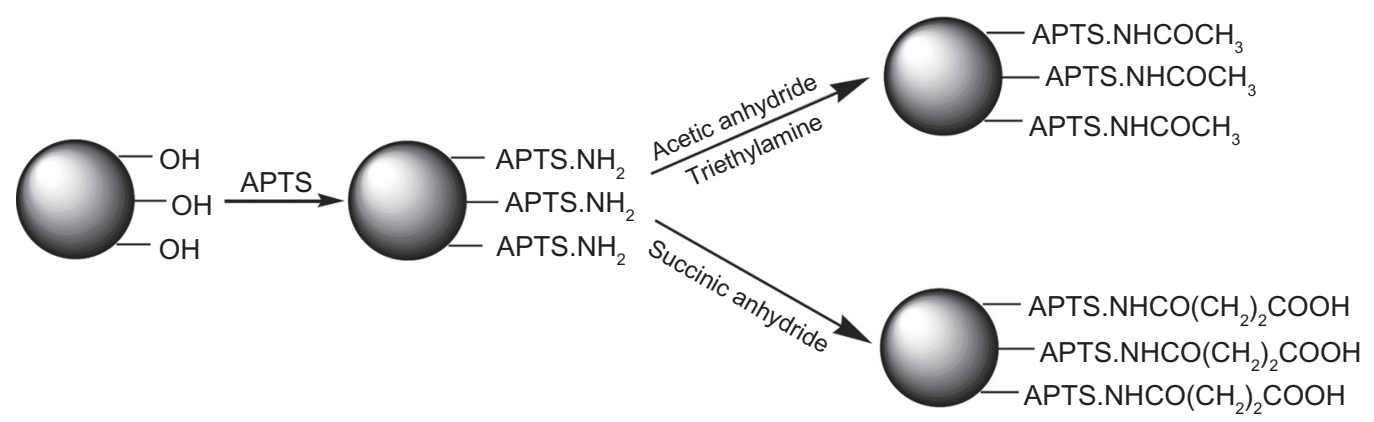

Scheme I Schematic representation of reactions to modify nanohydroxyapatite through aminopropyltriethoxysilane (APTS)-mediated functionalization. 
The metabolically active cells were detected by adding MTT to each well. The plates were then read at $570 \mathrm{~nm}$ using a microplate reader (MK3, Thermo Electron Corporation). The mean and standard deviation for the triplicate wells for each sample were reported.

The viability of L929 cells was also evaluated by visual observation of the morphology of cells treated with the pristine nanohydroxyapatite and the APTS-modified nanohydroxyapatite samples using a Leica DM IL LED inverted phase contrast microscope with a magnification of $200 \times$ for each sample.

\section{Hemolysis assay}

Human blood stabilized with heparin was kindly provided by Shanghai First People's Hospital (Shanghai, China). Human red blood cells were obtained by removing the serum via centrifugation and washing with phosphate-buffered solution five times according to the procedure reported in the literature. ${ }^{40}$ After that, the cells were diluted 10 times with phosphate-buffered solution. Then $0.2 \mathrm{~mL}$ of the diluted human red blood cell suspension was transferred to a $1.5 \mathrm{~mL}$ Eppendorf tube containing $0.8 \mathrm{~mL}$ of water, phosphatebuffered solution, or phosphate-buffered solution containing dispersed nanohydroxyapatite and its derivatives at different concentrations $(10,50,100,250$, and $500 \mu \mathrm{g} / \mathrm{mL})$, respectively. The above mixtures were then incubated at $37^{\circ} \mathrm{C}$ for 2 hours, followed by centrifugation (10000 rpm, 2 minutes), and the absorbance of the supernatants (hemoglobin) was recorded using a Perkin Elmer Lambda 25 ultraviolet-visible spectrometer.

\section{Statistical analysis}

One-way analysis of variance statistical analysis was performed to evaluate the significance of the toxicity of the nanohydroxyapatite and its derivatives. A $P$ value of 0.05 was considered as statistical significance level.

\section{Results and discussion}

In this study, we attempted to use APTS to silanize nanohydroxyapatite with abundant hydroxyl groups, resulting in the formation of n-HA-APTS, which possesses a large amount of primary amines on the surface. The surface amines of the n-HA-APTS particles were then acylated with acetic anhydride and succinic anhydride to form neutrally and negatively charged particles, respectively. The differently modified particles formed were extensively characterized using FTIR, ' $\mathrm{H}$ NMR, ICP-AES, TEM, X-ray diffraction, and zeta potential measurement.
FTIR spectroscopy was carried out to confirm APTSmediated modification on the nanohydroxyapatite particle surfaces (Figure 1). The typical absorption bands at $961 \mathrm{~cm}^{-1}$, $1032 \mathrm{~cm}^{-1}$, and $1088 \mathrm{~cm}^{-1}$ shown in Figure $1 \mathrm{~A}$ can be assigned to the characteristic bands of $\mathrm{PO}_{4}^{3-}$, and the weak peak at $3570 \mathrm{~cm}^{-1}$ corresponds to the stretching vibration of hydroxyl groups on the nanohydroxyapatite surface. Figure 1B shows the enlarged spectra with a wavenumber range of $1250 \mathrm{~cm}^{-1}$ to $1800 \mathrm{~cm}^{-1}$. The peak located at $1327 \mathrm{~cm}^{-1}$ for $\mathrm{n}-\mathrm{HA}-$ APTS is assigned to the $\mathrm{C}-\mathrm{N}$ stretching vibration band of APTS, suggesting successful modification of APTS on the surface of nanohydroxyapatite particles. Compared with the spectra for nanohydroxyapatite and n-HA-APTS, a new peak at $1636 \mathrm{~cm}^{-1}$ in the spectra of both n-HA-APTS.Ac and n-HA-APTS.SAH appears, which can be assigned to the $\mathrm{C}=\mathrm{O}$ stretch vibration band. The peak located at $1560 \mathrm{~cm}^{-1}$ in the spectra of n-HA-APTS, n-HA-APTS.Ac, and n-HA-APTS. $\mathrm{SAH}$ may be caused by moisture from the atmosphere. The peak located at $1480 \mathrm{~cm}^{-1}$ in the spectrum of n-HA-APTS can be assigned to the deformation modes of the amine groups that were strongly hydrogen-bonded with the silanol groups. ${ }^{41}$ The peaks at $1455 \mathrm{~cm}^{-1}$ and $1415 \mathrm{~cm}^{-1}$ may be attributed to $\mathrm{CO}_{2}$ adsorption on the surface of nanohydroxyapatite from the atmosphere. ${ }^{41}$ In general, the FTIR spectroscopy studies give relatively qualitative confirmation of APTS modification onto the nanohydroxyapatite surfaces; however, it is difficult to discern the difference between n-HA-APTS.Ac and n-HAAPTS.SAH samples.

The APTS-modified nanohydroxyapatite derivatives were further characterized by ${ }^{1} \mathrm{H}$ NMR spectroscopy (Figure 2). Pristine nanohydroxyapatite only displays the $-\mathrm{OH}$ proton signals at $3.17 \mathrm{ppm}$, as shown in Figure 2A. However, after modification with APTS and APTS-mediated further functionalization, the $-\mathrm{OH}$ proton signals at $3.17 \mathrm{ppm}$ almost disappear (Figure 2B-D). In addition, the $-\mathrm{CH}_{2}-$ proton signals (peak 1 at 0.47 ppm, peak 2 at 1.60 ppm, and peak 3 at $2.82 \mathrm{ppm}$ ) related to APTS can be clearly observed (Figure 2B). These results indicate successful modification of nanohydroxyapatite with APTS. Further reaction of APTS amines to form acetyl or carboxyl groups does not significantly alter the chemical shift of the above $3-\mathrm{CH}_{2}-$ proton signals of APTS. For the n-HA-APTS.Ac sample, the peak at 1.87 ppm (peak 4, Figure 2C) is related to the $-\mathrm{CH}_{3}$ protons of the acetyl groups,${ }^{42}$ confirming successful acetylation of APTS amines. For the n-HA-APTS.SAH sample, the peak at $2.30 \mathrm{ppm}$ (peak 4, Figure 2D) is assigned to the $-\mathrm{CH}_{2}-$ protons of the succinamic acid end groups, verifying the transformation of the APTS amines to carboxyl groups. 



Figure I Fourier transform infrared spectroscopy spectra of nanohydroxyapatite, n-HA-APTS, n-HA-APTS.Ac, and n-HA-APTS.SAH, respectively. (A) Wavenumber range of $650-4000 \mathrm{~cm}^{-1}$. (B) Wavenumber range of $1250-1800 \mathrm{~cm}^{-1}$.

Abbreviations: n-HA-APTS, nanohydroxyapatite-aminopropyltriethoxysilane; n-HA-APTS.Ac, neutralized nanohydroxyapatite-aminopropyltriethoxysilane; n-HA-APTS. SAH, negatively charged nanohydroxyapatite-aminopropyltriethoxysilane.

It is worth noting that a new peak at $2.1 \mathrm{ppm}$ can be clearly observed for all APTS-functionalized nanohydroxyapatite samples (Figure 2B-D), which may be associated with the silanol $-\mathrm{OH}$ proton signals of APTS that have not been reacted with the nanohydroxyapatite hydroxyl groups due to the much higher molar excess of the added APTS. To confirm this hypothesis further, the ${ }^{1} \mathrm{H}$ NMR spectra of APTS dissolved in both $\mathrm{CDCl}_{3}$ and $\mathrm{D}_{2} \mathrm{O}$ were compared (Figure $\mathrm{S} 1$, Supplementary material). The ${ }^{1} \mathrm{H}$ NMR spectrum of APTS in $\mathrm{CDCl}_{3}$ is different from that of APTS dissolved in $\mathrm{D}_{2} \mathrm{O}$, because APTS can be hydrolyzed in $\mathrm{D}_{2} \mathrm{O}$ to form silanol groups. It is clear that the proton signal of $-\mathrm{CH}_{2}-$ adjacent to Si shifts to the high field after hydrolysis, and a new peak at about $2.2 \mathrm{ppm}$ assigned to the $-\mathrm{OH}$ proton signal of silanol appears. Therefore, the peak at $2.1 \mathrm{ppm}$ in Figure 2B-D is likely associated with the hydrolyzed APTS silanol $-\mathrm{OH}$ proton signals. The much more molar excess of APTS added to the pristine nanohydroxyapatite samples may not allow complete reaction of the APTS silanol groups with the hydroxyl groups of nanohydroxyapatite.

In order to quantify the amount of APTS modified onto the nanohydroxyapatite particles, ICP-AES was carried out to analyze the elemental composition of the n-HA-APTS sample. The mass fractions of calcium and silicon were determined to be $382.8 \mu \mathrm{g} / \mathrm{mg}$ and $14.3 \mu \mathrm{g} / \mathrm{mg}$, respectively. With the known mass fractions of calcium and silicon, the amount of modified APTS can be calculated according to the following equation:

$$
\begin{aligned}
\omega_{A P T S} & =\frac{m_{A P T S}}{m_{n-H A}+m_{A P T S}} \\
& =\frac{\left(\omega_{s i} / M_{s i}\right) \cdot M_{A P T S}^{\prime}}{\left(\omega_{c a} / 10 M_{c a}\right) \cdot M^{\prime}{ }_{n-H A}+\left(\omega_{s i} / M_{s i}\right) \cdot M_{A P T S}^{\prime}}
\end{aligned}
$$

where $\omega_{A P T S}$ is the mass percentage of APTS modified onto nanohydroxyapatite particles, $m_{n-H A}$ and $m_{A P T S}$ are the masses of nanohydroxyapatite and APTS in the n-HA-APTS sample, 

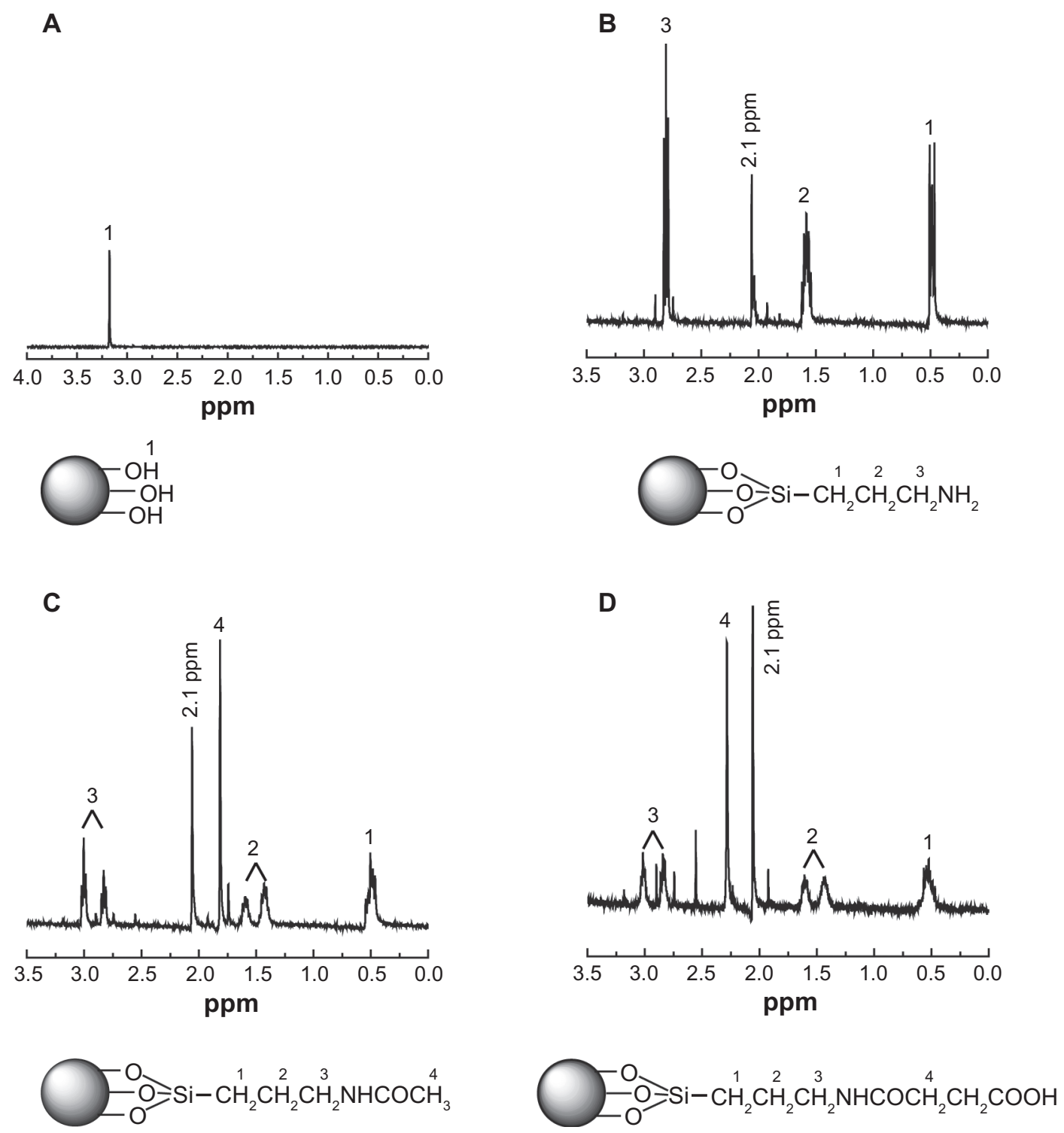

Figure 2 'H Nuclear magnetic resonance spectra of nanohydroxyapatite (A), nanohydroxyapatite-aminopropyltriethoxysilane (B), neutralized nanohydroxyapatiteaminopropyltriethoxysilane (C), and negatively charged nanohydroxyapatite-aminopropyltriethoxysilane (D), respectively.

respectively, $\omega_{s i}$ and $\omega_{c a}$ are the mass fractions of silicon and calcium in the n-HA-APTS sample, respectively, and $M_{s i}$ and $M_{c a}$ are the atomic weights of silicon and calcium, respectively. It is worth noting that $M_{s i}^{\prime}$ and $M_{n-H A}^{\prime}$ in the equation stands for the incoming molecular mass of APTS and nanohydroxyapatite after APTS modification reaction to form n-HA-APTS, ${ }^{43}$ where the value of $M_{s i}^{\prime}$ and $M_{n-H A}^{\prime}$ is calculated to be 134 and 953, respectively. The mass of APTS reacted with nanohydroxyapatite was estimated to be $6.96 \%$ according to Equation 1.

The morphology of the nanohydroxyapatite particles before and after different modifications was observed by TEM (Figure 3). It is clear that the pristine nanohydroxyapatite particles have a regular rod shape with a diameter and length of $37 \pm 9 \mathrm{~nm}$ and $118 \pm 42 \mathrm{~nm}$, respectively (Figure 3A). The modification of nanohydroxyapatite particles with APTS and further acetylation or carboxylation of the APTS amines (Figure 3B-D) seem not to alter the morphology of the particles significantly when compared with pristine nanohydroxyapatite particles. No aggregation was observed for all samples dispersed in aqueous solution, suggesting that APTS is uniformly coated onto each nanohydroxyapatite particle even after further APTS amine functionalization to form acetyl or carboxyl groups. The somewhat aggregated particles shown in the TEM images of all samples are presumably due to the TEM sample preparation method. The air-drying of the aqueous suspension of the samples before TEM measurement may lead to a partial aggregation or interconnection of particles, in agreement with previous literature. ${ }^{44}$ 




Figure 3 Transmission electron microscopic images of nanohydroxyapatite (A), nanohydroxyapatite-aminopropyltriethoxysilane(B), neutralized nanohydroxyapatiteaminopropyltriethoxysilane (C), and negatively charged nanohydroxyapatiteaminopropyltriethoxysilane (D), respectively.

The crystalline structure of the pristine nanohydroxyapatite particles and the different APTS-modified nanohydroxyapatite particles were investigated by X-ray diffraction (Figure 4). The diffraction peaks of the pristine nanohydroxyapatite particles were indexed in curve A (Joint Committee for Powder Diffraction Standards standard X-ray diffraction data for pure hydroxyapatite: 09-0432). It is clear that after grafting with APTS and further acetylation or carboxylation of APTS amines, the X-ray diffraction patterns remain similar to that of the pristine nanohydroxyapatite particles, suggesting that APTS modification reactions did not have any appreciable effect on the original crystal structure of the nanohydroxyapatite particles.

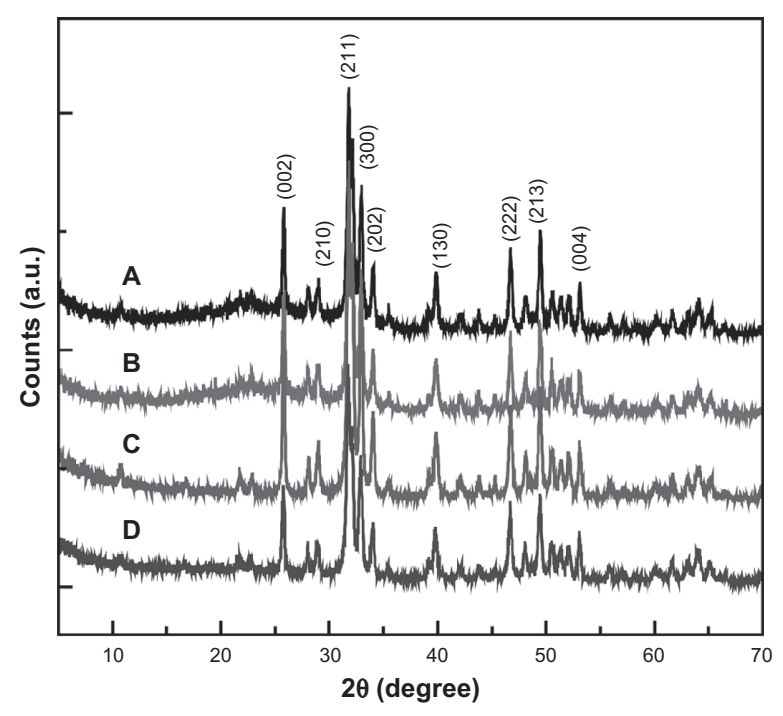

Figure 4 X-ray diffraction patterns of nanohydroxyapatite $(A)$, nanohydroxyapatiteaminopropyltriethoxysilane (B), neutralized nanohydroxyapatite-aminopropyltriethoxysilane (C), and negatively charged nanohydroxyapatite-aminopropyltriethoxysilane (D), respectively.
The surface modification of nanohydroxyapatite was finally assessed by zeta potential measurement. The surface potential of the pristine nanohydroxyapatite $(-8.22 \pm 0.08 \mathrm{mV})$ was reversed to be positive $(7.14 \pm 0.27 \mathrm{mV})$ after modification of APTS possessing primary amine groups. Further acetylation of the APTS amines neutralized the positive charges of the n-HA-APTS particles $(1.31 \pm 0.08 \mathrm{mV})$, whereas carboxylation of the APTS amines of n-HA-APTS was able to reverse further the positive charge of nanohydroxyapatite to be highly negative $(-21.8 \pm 0.49 \mathrm{mV})$. Overall, FTIR, ${ }^{1} \mathrm{H}$ NMR, ICP-AES, TEM, X-ray diffraction, and zeta potential measurement confirmed successful surface modification of nanohydroxyapatite particles with APTS and APTS derivatives.

The cytotoxicity of nanohydroxyapatite and its derivatives was tested by MTT viability assay of L929 cells after treatment with nanohydroxyapatite and its derivatives. As shown in Figure 5, there was no statistically significant difference in cell viability for L929 cells between the control cells treated with phosphate-buffered solution, and the cells treated with the pristine nanohydroxyapatite, the n-HA-APTS.Ac, and the n-HA-APTS.SAH particles at the concentration range of $0-100 \mu \mathrm{g} / \mathrm{mL}(P>0.05)$. However, there is a statistically significant difference in cell viability between the phosphate-buffered solution control and the n-HA-APTS particles at different concentrations $(P<0.05$ at 1 and $10 \mu \mathrm{g} / \mathrm{mL}, P<0.01$ at $100 \mu \mathrm{g} / \mathrm{mL}$ ), implying that the modified APTS on the nanohydroxyapatite surface without neutralization of the amine groups inhibits cell

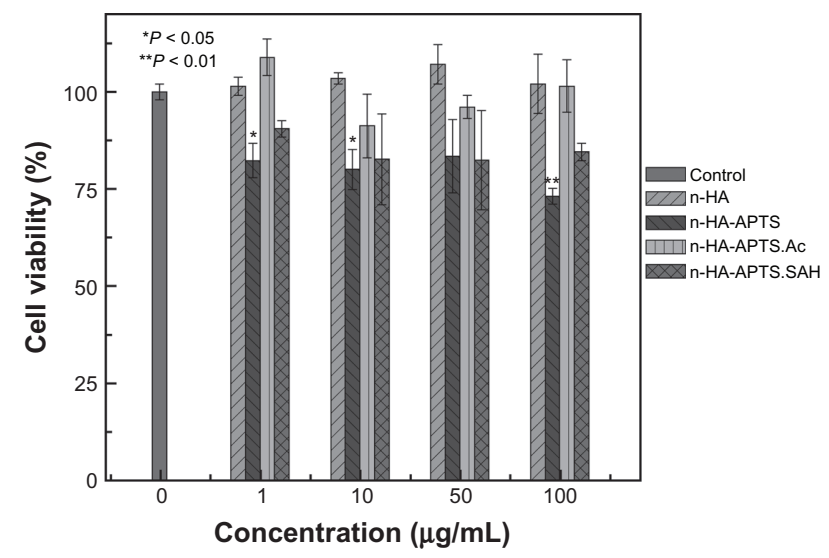

Figure 5 MTT viability assay of L929 cells after treatment with nanohydroxyapatite particles and aminopropyltriethoxysilane-modified nanohydroxyapatite derivatives for 24 hours.

Notes: Mean and standard deviation for triplicate wells were reported. Data are expressed as the mean \pm standard deviation. Statistical significance was calculated using analysis of variance. $* P<0.05$; $* * P<0.01$.

Abbreviations: n-HA-APTS, nanohydroxyapatite-aminopropyltriethoxysilane; n-HA-APTS.Ac, neutralized nanohydroxyapatite-aminopropyltriethoxysilane; n-HAAPTS.SAH, negatively charged nanohydroxyapatite-aminopropyltriethoxysilane. 


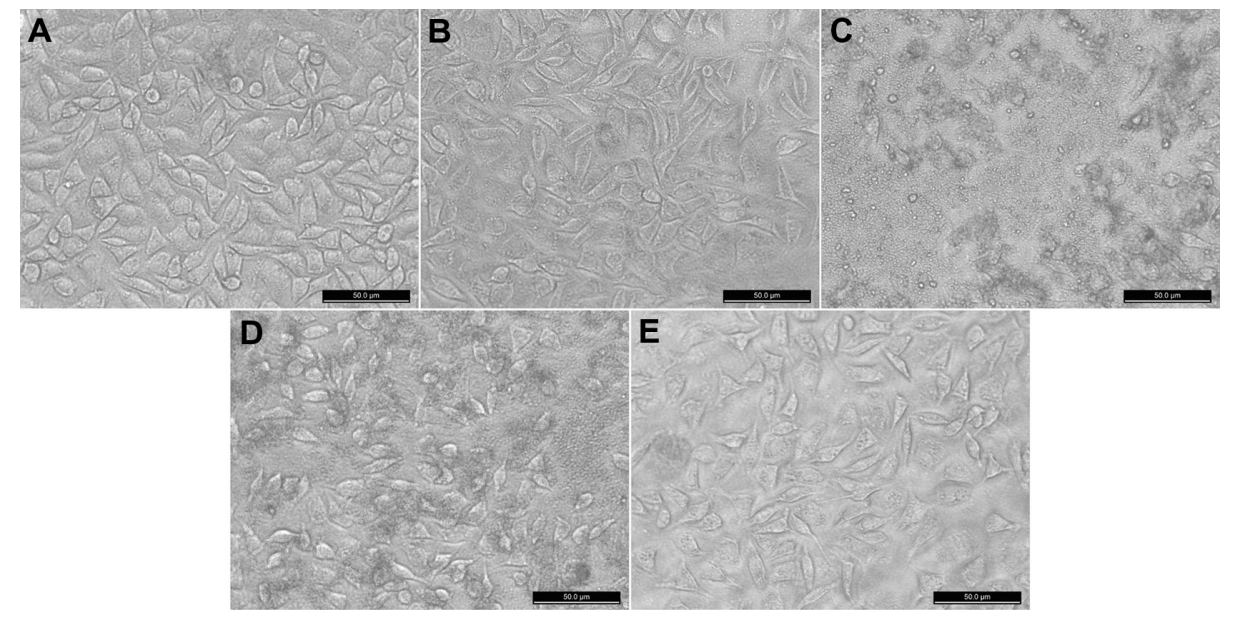

Figure 6 Phase-contrast photomicrograph of (A) untreated L929 cells (phosphate-buffered control) and L929 cells treated with I00 $\mu$ g/mL of (B) nanohydroxyapatite, (C) nanohydroxyapatite-aminopropyltriethoxysilane, (D) neutralized nanohydroxyapatite-aminopropyltriethoxysilane, and (E) negatively charged nanohydroxyapatiteaminopropyltriethoxysilane for 24 hours, respectively.

growth to some extent. This also suggests that the APTS amine-induced cytotoxicity of nanohydroxyapatite particles can be readily reduced by acetylating or carboxylating of the APTS amine groups. These results are consistent with our previous report. ${ }^{39}$ The relatively toxic APTS-modified nanohydroxyapatite particles with surface amine groups should be due to the electrostatic interaction between the positively charged particles and the negatively charged cell membranes. ${ }^{45-47}$ The neutralization of the surface amine groups to form acetamide or carboxyl groups can significantly

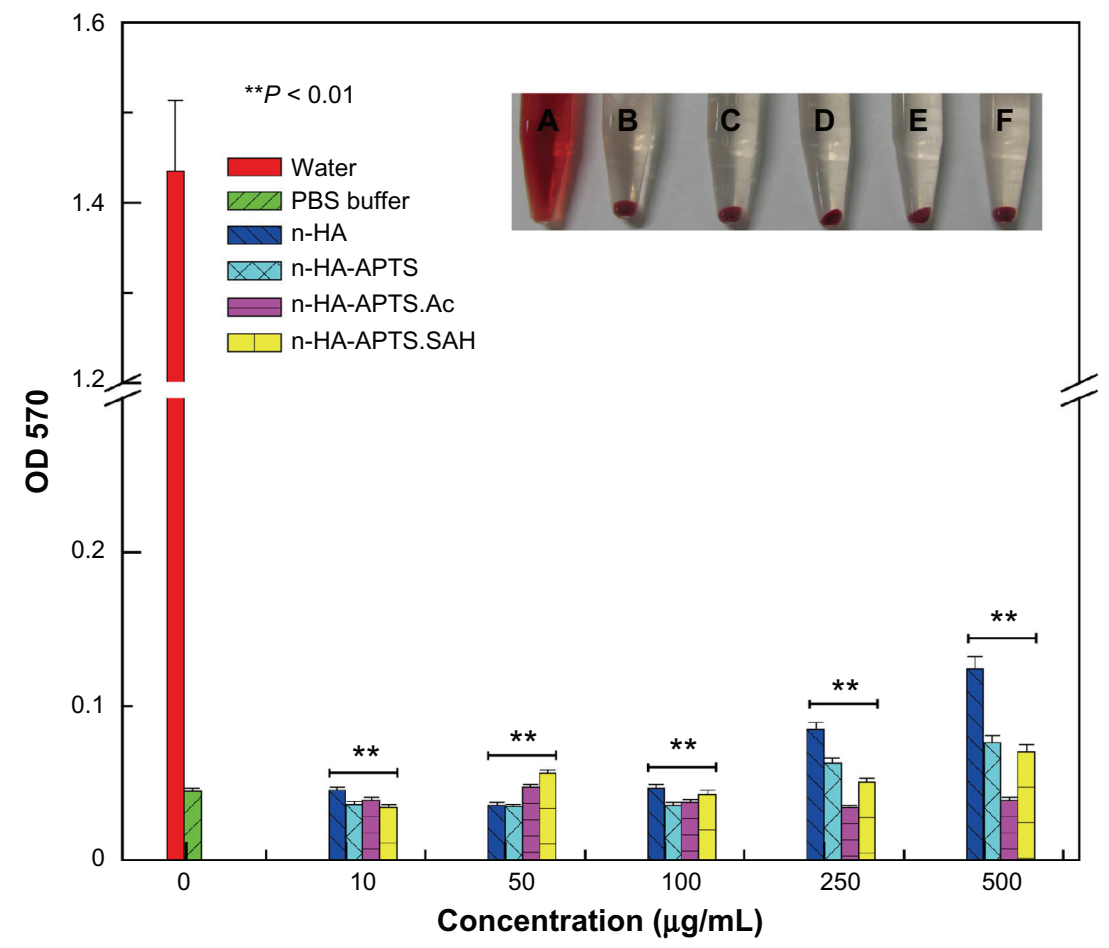

Figure 7 Hemolytic assay of human red blood cells after treatment with distilled water, phosphate-buffered solution, and differently functionalized nanohydroxyapatite derivatives for 24 hours. The mean and standard deviation for triplicate samples were reported. The inset shows a photograph of human red blood cells exposed to (A) distilled water, (B) phosphate-buffered solution, (C) nanohydroxyapatite, (D) n-HA-APTS, (E) n-HA-APTS.Ac, and (F) n-HA-APTS.SAH for 2 hours (sample concentration $500 \mu \mathrm{g} / \mathrm{mL})$, followed by centrifugation.

Note: Statistical significance was calculated using analysis of variance. $* * p<0.01$.

Abbreviations: n-HA-APTS, nanohydroxyapatite-aminopropyltriethoxysilane; n-HA-APTS.Ac, neutralized nanohydroxyapatite-aminopropyltriethoxysilane; n-HA-APTS. $\mathrm{SAH}$, negatively charged nanohydroxyapatite-aminopropyltriethoxysilane. 
reduce the electrostatic interaction between the particles and the cells, thereby improving the biocompatibility of APTSmodified nanohydroxyapatite particles. ${ }^{39}$

The cytotoxicity of nanohydroxyapatite and its derivatives was further confirmed by phase contrast microscopic visualization of cell morphology changes after treatment with nanohydroxyapatite with different surface functionalities for 24 hours. Figure 6 shows the morphology of untreated L929 cells (Figure 6A), L929 cells treated with pristine nanohydroxyapatite particles (Figure 6B), n-HA-APTS particles (Figure 6C), n-HA-APTS.Ac particles (Figure 6D), and n-HA-APTS.SAH particles (Figure 6E) at a concentration of $100 \mu \mathrm{g} / \mathrm{mL}$. It is clear that the pristine nanohydroxyapatite particles, n-HA-APTS.Ac particles with neutral surface charge, and n-HA-APTS.SAH particles with a negative surface charge do not induce appreciable cell morphology changes when compared with untreated L929 cells, confirming their excellent biocompatibility. In contrast, when L929 cells were treated with positively charged n-HA-APTS particles, a portion of cells became rounded and nonadherent, indicating that the cells had undergone apoptosis. These results are consistent with the MTT assay data.

For practical biomedical applications, especially in circumstances where nanoparticles need to be intravenously injected, blood compatibility becomes a major concern for the utility of materials. Blood compatibility of nanohydroxyapatite and its derivatives was evaluated by in vitro hemolysis assay (Figure 7). No obvious hemolysis phenomenon was observed after exposure of nanohydroxyapatite and its derivatives with different surface functionalities to human red blood cells, even when the sample concentration is up to $500 \mu \mathrm{g} / \mathrm{mL}$ (inset of Figure 7). The hemolytic effects of each sample at different concentrations were further determined by recording absorbance of the supernatant at $570 \mathrm{~nm}$ using ultraviolet-visible spectroscopy. A significant difference $(P<0.01)$ in absorbance at $570 \mathrm{~nm}$ associated with hemoglobin was found between the control group (human red blood cells exposed to distilled water) and the experimental groups. This indicates that, in the concentration range studied, nanohydroxyapatite and its derivatives with different functionalities do not show any appreciable hemolytic effect, implying that the surface modification of nanohydroxyapatite via APTS-mediated functionalization does not compromise the compatibility between pristine nanohydroxyapatite particles and blood.

\section{Conclusion}

In summary, a simple approach was developed to modify nanohydroxyapatite particles via an APTS-mediated chemical reaction. The silanization of nanohydroxyapatite particles with APTS creates particles with abundant primary amines on their surfaces, which can be further acetylated or carboxylated to make nanohydroxyapatite particles with a neutral surface charge or negative surface charge, respectively. We showed that pristine nanohydroxyapatite particles, neutral (n-HA-APTS.Ac), and negatively charged (n-HA-APTS. $\mathrm{SAH}$ ) n-HA derivatives do not display cytotoxicity at a concentration up to $100 \mu \mathrm{g} / \mathrm{mL}$. However, without further modification, APTS-modified nanohydroxyapatite particles are slightly cytotoxic at the concentrations studied. The hemolytic effect of pristine nanohydroxyapatite particles is not compromised after APTS-mediated surface functionalization. The significance of our study is that through APTS modification and further functionalization, the surface charge of nanohydroxyapatite particles can be regulated to be positive, neutral, and negative with different functional groups. We anticipate that APTS-functionalized nanohydroxyapatite with reactive surface functional groups may be beneficial for further conjugation with other polymers or biological ligands onto the particle surfaces for various biomedical applications.

\section{Acknowledgments}

This research is financially supported by the National Basic Research Program of China (973 Program, 2007CB936000, 2011CB510106, 2011CB504300), the National Natural Science Foundation of China (20974019, 81101150, 81071747), the Program for Professor of Special Appointment (Eastern Scholar) at Shanghai Institutions of Higher Learning (XS and JW), the Innovation Funds of Donghua University Doctorate Dissertation of Excellence (BC201107), and the Fundamental Research Funds for the Central Universities (MS, RG, XC, XS). XS gratefully acknowledges the Fundação para a Ciência e a Tecnologia and Santander bank for the Chair in Nanotechnology.

\section{Disclosure}

The authors report no conflicts of interest relevant to this research.

\section{References}

1. Fang X, Ma H, Xiao S, et al. Facile immobilization of gold nanoparticles into electrospun polyethyleneimine/polyvinyl alcohol nanofibers for catalytic applications. J Mater Chem. 2011;21:4493-4501.

2. Daniel MC, Astruc D. Gold nanoparticles: Assembly, supramolecular chemistry, quantum-size-related properties, and applications toward biology, catalysis, and nanotechnology. Chem Rev. 2004;104:293-346.

3. Luo X, Morrin A, Killard AJ, Smyth MR. Application of nanoparticles in electrochemical sensors and biosensors. Electroanalysis. 2006;18:319-326. 
4. Xu JJ, Luo XL, Du Y, Chen HY. Application of $\mathrm{MnO}_{2}$ nanoparticles as an eliminator of ascorbate interference to amperometric glucose biosensors. Electrochem Commun. 2004;6:1169-1173.

5. Gao J, Gu H, Xu B. Multifunctional magnetic nanoparticles: Design, synthesis, and biomedical applications. Acc Chem Res. 2009;42:1097-1107.

6. Shen M, Shi X. Dendrimer-based organic/inorganic hybrid nanoparticles in biomedical applications. Nanoscale. 2010;2:1596-1610.

7. Wang H, Zheng L, Peng C, et al. Computed tomography imaging of cancer cells using acetylated dendrimer-entrapped gold nanoparticles. Biomaterials. 2011;32:2979-2988.

8. Yang H, Zhang C, Shi X, et al. Water-soluble superparamagnetic manganese ferrite nanoparticles for magnetic resonance imaging. Biomaterials. 2010;31:3667-3673.

9. Ito A, Takizawa $\mathrm{Y}$, Honda $\mathrm{H}$, et al. Tissue engineering using magnetite nanoparticles and magnetic force: Heterotypic layers of cocultured hepatocytes and endothelial cells. Tissue Eng. 2004;10:833-840.

10. Tasker LH, Sparey-Taylor GJ, Nokes LDM. Applications of nanotechnology in orthopaedics. Clin Orthop Relat Res. 2007;456:243-249.

11. Hou CH, Hou SM, Hsueh YS, Lin J, Wu HC, Lin FH. The in vivo performance of biomagnetic hydroxyapatite nanoparticles in cancer hyperthermia therapy. Biomaterials. 2009;30:3956-3960.

12. Shi Z, Huang X, Cai Y, Tang R, Yang D. Size effect of hydroxyapatite nanoparticles on proliferation and apoptosis of osteoblast-like cells. Acta Biomater. 2009;5:338-345.

13. Hornez JC, Chai F, Monchau F, Blanchemain N, Descamps M, Hildebrand HF. Biological and physico-chemical assessment of hydroxyapatite (HA) with different porosity. Biomol Eng. 2007;24:505-509.

14. Zhu SH, Huang BY, Zhou KC, et al. Hydroxyapatite nanoparticles as a novel gene carrier. J Nanopart Res. 2004;6:307-311.

15. Fabbri P, Bondioli F, Messori M, Bartoli C, Dinucci D, Chiellini F. Porous scaffolds of polycaprolactone reinforced with in situ generated hydroxyapatite for bone tissue engineering. J Mater Sci Mater Med. 2010;21:343-351

16. Zhang C, Li C, Huang S, et al. Self-activated luminescent and mesoporous strontium hydroxyapatite nanorods for drug delivery. Biomaterials. 2010;31:3374-3383.

17. Petit R. The use of hydroxyapatite in orthopaedic surgery: a ten-year review. Eur J Orthop Surg Traumatol. 1999;9:71-74.

18. Cooke FW. Ceramics in orthopedic surgery. Clin Orthop Relat Res. 1992;276:135-146.

19. Akao M, Aoki H, Kato K. Mechanical properties of sintered hydroxyapatite for prosthetic applications. J Mater Sci. 1981;16:809-812.

20. Lopes MA, Monteiro FJ, Santos JD. Glass-reinforced hydroxyapatite composites: fracture toughness and hardness dependence on microstructural characteristics. Biomaterials. 1999;20:2085-2090.

21. Cengiz B, Gokce Y, Yildiz N, Aktas Z, Calimli A. Synthesis and characterization of hydroxyapatite nanoparticles. Colloids Surf A Physicochem Eng Asp. 2008;322:29-33.

22. Wei G, Ma PX. Structure and properties of nano-hydroxyapatite/ polymer composite scaffolds for bone tissue engineering. Biomaterials. 2004;25:4749-4757.

23. Aoki H, Kutsuno T, Li W, Niwa M. An in vivo study on the reaction of hydroxyapatite-sol injected into blood. J Mater Sci Mater Med. 2000;11:67-72.

24. Peng J, Li X, Guo G, et al. Preparation and characterization of poly (vinyl alcohol)/poly(-caprolactone)-poly(ethylene glycol)-poly (-caprolactone)/nano-hydroxyapatite composite membranes for tissue engineering. J Nanosci Nanotechnol. 2011;11:2354-2360.

25. Deng C, Yang XB, Weng J. Fabrication and in vitro bioactivity of poly ( $\varepsilon$-caprolactone) composites filled with silane-modified nano-apatite particles. Int J Polym Mater. 2011;60:374-383.

26. Seyedjafari E, Soleimani M, Ghaemi N, Shabani I. Nanohydroxyapatitecoated electrospun poly (l-lactide) nanofibers enhance osteogenic differentiation of stem cells and induce ectopic bone formation. Biomacromolecules. 2010;11:3118-3125.
27. Kim HW, Lee HH, Knowles JC. Electrospinning biomedical nanocomposite fibers of hydroxyapatite/poly (lactic acid) for bone regeneration. J Biomed Mater Res A. 2006;79:643-649.

28. Xiao F, Ye J, Wang Y, Rao P. Deagglomeration of HA during the precipitation synthesis. J Mater Sci. 2005;40:5439-5442.

29. Zhang R, Ma PX. Poly ( $\alpha$-hydroxyl acids)/hydroxyapatite porous composites for bone-tissue engineering. I. Preparation and morphology. J Biomed Mater Res A. 1999;44(4):446-455.

30. Zuo Y, Li Y, Li J, et al. Novel bio-composite of hydroxyapatite reinforced polyamide and polyethylene: Composition and properties. Mater Sci Eng A Struct Mater. 2007;452-453:512-517.

31. Zhang Y, Venugopal JR, El-Turki A, Ramakrishna S, Su B, Lim CT. Electrospun biomimetic nanocomposite nanofibers of hydroxyapatite/chitosan for bone tissue engineering. Biomaterials. 2008;29:4314-4322.

32. Tang T, Fan H, Ai S, Han R, Qiu Y. Hemoglobin (Hb) immobilized on amino-modified magnetic nanoparticles for the catalytic removal of bisphenol A. Chemosphere. 2011;83:255-264.

33. Song Y, Hildebrand H, Schmuki P. Photoinduced release of active proteins from $\mathrm{TiO}_{2}$ surfaces. Electrochem Commun. 2009;11: 1429-1433.

34. Pan B, Gao F, Gu H. Dendrimer modified magnetite nanoparticles for protein immobilization. J Colloid Interface Sci. 2005;284:1-6.

35. Ma M, Zhang Y, Yu W, Shen HY, Zhang H, Gu N. Preparation and characterization of magnetite nanoparticles coated by amino silane. Colloids Surf A Physicochem Eng Asp. 2003;212:219-226.

36. Song YY, Hildebrand H, Schmuki P. Optimized monolayer grafting of 3-aminopropyltriethoxysilane onto amorphous, anatase and rutile $\mathrm{TiO} 2$. Surf Sci. 2010;604:346-353.

37. Balasundaram G, Sato M, Webster TJ. Using hydroxyapatite nanoparticles and decreased crystallinity to promote osteoblast adhesion similar to functionalizing with RGD. Biomaterials. 2006;27: 2798-2805.

38. Bauer F, Gläel H, Ulrich D, Hartmann E, Sauerland V, Mehnert R. Trialkoxysilane grafting onto nanoparticles for the preparation of clear coat polyacrylate systems with excellent scratch performance. Progress in Organic Coatings. 2003;47:147-153.

39. Shen M, Wang SH, Shi X, et al. Polyethyleneimine-mediated functionalization of multiwalled carbon nanotubes: synthesis, characterization, and in vitro toxicity assay. J Phys Chem C. 2009;113:3150-3156.

40. Wu H, Liu G, Zhuang Y, et al. The behavior after intravenous injection in mice of multiwalled carbon nanotube/Fe (3) O (4) hybrid MRI contrast agents. Biomaterials. 2011;32:4867-4876.

41. Joschek S, Nies B, Krotz R, Göferich A. Chemical and physicochemical characterization of porous hydroxyapatite ceramics made of natural bone. Biomaterials. 2000;21:1645-1658.

42. Gabrielson NP, Daniel W. Acetylation of polyethylenimine enhances gene delivery via weakened polymer/DNA interactions. Biomacromolecules. 2006;7:2427-2435.

43. Prado AGS, Airoldi C. Immobilization of the pesticide 2,4-dichlorophenoxyacetic acid on a silica gel surface. Pest Manag Sci. 2000;56:419-424.

44. Shi X, Briseno AL, Sanedrin RJ, Zhou F. Formation of uniform polyaniline thin shells and hollow capsules using polyelectrolytecoated microspheres as templates. Macromolecules. 2003;36: 4093-4098.

45. Hong S, Bielinska AU, Mecke A, et al. Interaction of poly (amidoamine) dendrimers with supported lipid bilayers and cells: hole formation and the relation to transport. Bioconjug Chem. 2004;15:774-782.

46. Hong S, Leroueil PR, Elizabeth $\mathrm{K}$, et al. Interaction of polycationic polymers with supported lipid bilayers and cells: nanoscale hole formation and enhanced membrane permeability. Bioconjug Chem. 2006;17:728-734.

47. Shi X, Wang S, Sun H, Baker JR Jr. Improved biocompatibility of surface functionalized dendrimer-entrapped gold nanoparticles. Soft Matter. 2007;3:71-74. 


\section{Supplementary material}


Figure SI ' $\mathrm{H}$ nuclear magnetic resonance spectra of aminopropyltriethoxysilane (APTS) dissolved in $\mathrm{CDCl}_{3}(\mathbf{A})$ and $\mathrm{D}_{2} \mathrm{O}(\mathbf{B})$.

International Journal of Nanomedicine

\section{Publish your work in this journal}

The International Journal of Nanomedicine is an international, peerreviewed journal focusing on the application of nanotechnology in diagnostics, therapeutics, and drug delivery systems throughout the biomedical field. This journal is indexed on PubMed Central, MedLine, CAS, SciSearch ${ }^{\circledR}$, Current Contents ${ }^{\circledR} /$ Clinical Medicine,

\section{Dovepress}

Journal Citation Reports/Science Edition, EMBase, Scopus and the Elsevier Bibliographic databases. The manuscript management system is completely online and includes a very quick and fair peer-review system, which is all easy to use. Visit http://www.dovepress.com/ testimonials.php to read real quotes from published authors. 